\title{
Myofibroblastoma tumour of the breast
}

\author{
S. Raviraj \\ University Surgical Unit, Teaching Hospital, Jaffna, Sri Lanka
}

Keywords: Metaplastic carcinoma; immunohistochemical assay; myofibroblastoma (mammary type); solitary fibrous tumour; sarcoma

\section{Introduction}

Myofibroblastomas are an unusual benign tumour of the breast predominantly affecting elderly males in their sixth to seventh decade. Recently, quite a few cases have been reported in women [3]. Myofibroblastoma are unusual rapidly growing benign mesenchymal tumours of the breast. These tumours tend to share morphological and immunohistochemical features with solitary fibrous tumours. Myofibroblastomas are typically a bland-looking spindle cell tumour exhibiting expression of vimentin, desmin and CD34 in most cases [5]. The term myofibroblastoma was first coined by Toker et al in 1981 to indicate a bland-looking spindle cell neoplasm with a variable myofibroblastic differentiation [7], and later another case was reported by Wargotzin in 1987 [3].

\section{Case Report}

A 72 year old previously healthy male presented to the surgical clinic with a lump in the right breast for a duration of one month. He had noticed a painless lump in the right breast which was rapidly growing, but had no other complaints. Clinical examination revealed a $3 \mathrm{~cm} \times 2 \mathrm{~cm}$ non-tender breast lesion with ill-defined margins that was not attached to the skin or deeper structures. There was no local regional lymph node enlargement in the axilla or supraclavicular region. The opposite breast and axilla were normal. Clinically it was suspected to be a malignant lesion. Ultrasound scan revealed a suspected malignant lesion in the upper medial quadrant of the right breast measuring $2 \mathrm{~cm} \times 1 \mathrm{~cm}$, and ultrasound-guided fine needle aspiration revealed the possibility of a metaplastic carcinoma. The pathologist recommended an excision biopsy for this patient. Investigations such as a chest X-ray PA, an ultrasound abdomen and a thoraco-lumbar X-ray were done in this patient, but did not reveal any metastatic deposits.

A multi-disciplinary team discussion was carried out and it was decided to proceed with a wide-local excision of the lump

Correspondence: S. Raviraj

E-mail: dr.s.raviraj@gmail.com

DOI: http://10.4038/sljs.v34i2.8268

\section{(c) BY}

The Sri Lanka Journal of Surgery 2016; 34(2): 30-31 with adequate margins as the first option, and further management to be decided based on the biopsy report. The patient was informed of the procedure and surgery was carried out without any post-operative complications.

\section{Biopsy report}

Macroscopically, a circumscribed tan coloured tumour measuring $20 \mathrm{~mm} \times 20 \mathrm{~mm}$, situated $5 \mathrm{~mm}$ away from the deep resection margin, $15 \mathrm{~mm}$ away from the inferior resection margin and $30 \mathrm{~mm}$ away from the superior resection margin, was revealed.

Microscopic section revealed a circumscribed lesion composed of spindle shaped cells which were arranged haphazardly in fascicles. The cells had elongated nuclei with eosinophilic cytoplasm. The mitotic count was 3/10 high power field. The tumour contained hyalinized collagen bundles, scattered mast cells, and adipocytes concentrated in the periphery. There was no evidence of lymphovascular, perineural invasion or tumour necrosis. The final comments by the histopathologist was that the excised tumour was 'compatible with a mammary type of myofibroblastoma and suggested immunohistochemical marking'.

\section{Immunohistochemical assay of Cd34}

The tumour cells stained strongly positive for CD34 and focally positive for smooth muscle actin (SMA). Occasionally, cells stained positively for epithelial membrane antigen (EMA). The tumour cells stained negatively for cytokeratin and BCL-2.

The conclusion of histopathology and immunohistochemistry reports was that the excised tumour had morphological and immune-phenotypic features compatible with a mammary type myofibroblastoma, which is a benign breast condition in this patient. The patient was followed up at the surgical clinic without any complications after the wide-local excision.

\section{Discussion}

In this case report we present an unusual breast tumour which wass clinically and ultrasonically suspicious of a malignancy, with a false FNAC diagnosis of metaplastic carcinoma or sarcoma. FNAC or core biopsy of a myofibroblastoma can be difficult to interpret especially when one is faced with an unusual variant [5-7].

There are several morphological variants of myofibroblastomas which includes cellular, infiltrating, 
epithelioid-like, lipomatous, colonized and myxoid [8]. Immunohistochemical analysis helps in confirming the diagnosis of these cases. Most cases of myofibroblastomas are typically positive to $\mathrm{CD} 34$, vimentin and desmin. Cytokeratin, EMA, S-100 protein and CD117 are consistently negative [8].

\section{Management}

The FNAC and core needle biopsy provided limited specimen that unfortunately contained only carcinoma like cellular areas which in most cases is suspicious of a malignant lesion or myofibroblastoma of the breast, and therefore the pathologist suggested an excision biopsy of the lesion. Myofibroblastoma is a benign condition and hence further management only requires follow-up as an outpatient in a surgical clinic without any interferences. If the report revealed a malignant lesion of the breast it should be managed under the protocol of breast carcinoma after multidisciplinary team discussion.

\section{References}

1. Arsenovic N, Abdullah KE, Shamin KS. Mammary type of
Myofibroblastoma of soft tissue. Indian J Pathol Microbiol.2001; 54:391-3.

http://dx.doi.org/10.4103/0377-4929.81646

2. Mele M, Jensen V, Wronecki A, Lelkatis G. Myofibroblastma of the breast: case report and literature review. Int J Surg Case Rep. 2011;2: 93-6.

http://dx.doi.org/10.1016/j.ijscr.2011.02.006

3. Rafique A, Arshad A. Myofibroblastoma: an unusual rapidly growing benign tumour in a male breast. Journal of the College of Physicians and Surgeons Pakistan. 2013; vol. 23; 11:818-19

4. Desrosiers I, Rezks, Larkin A. Myofibroblastoma of the breast. Histopathology. 2007; 51: 568-72. http://dx.doi.org/10.1111/j.1365-2559.2007.02808.x

5. Margo G. Epithelial cell Myofibroblastoma of the breast: a potential diagnostic pitfall. Breast J. 2012; 18: 278-279. http://dx.doi.org/10.1111/j.1524-4741.2011.01172.x

6. Margo G. Epithelial cell Myofibriblastoma of the Breast expanding the morphologic spectrum. Am J Sury Pathol. 2009; $33 ; 1085-1092$

http://dx.doi.org/10.1097/PAS.0b013e31819e642a

7. Margo G. Mammary Myofibroblastoma: a tumour with wide morphologic spectrum. Arch Pathol Lab. 2008. Med-vol 1323.

\section{Key Points:}

- The incidence of myofibroblastomas has increased in recent years most likely due to an increase in clinical assessment and radiological screening.

- This unusual benign tumour may represent a potential diagnostic pitfall especially when interpreting with fine needle aspiration or core biopsy.

- It is a challenge for pathologists to be aware of the wide morphological spectrum exhibited by myofibroblastomas to avoid the misdiagnosis of malignancy. 